\title{
A Single Arm, Phase II Study of Simvastatin Plus XELOX and Bevacizumab as First-Line Chemotherapy in Metastatic Colorectal Cancer Patients
}

\author{
Youjin Kim, MD',2 \\ Tae Won Kim, MD, PhD ${ }^{3}$ \\ Sae Won Han, MD, PhD ${ }^{4}$ \\ Joong Bae Ahn, MD, PhD 5 \\ Seung Tae Kim, MD, PhD ${ }^{1}$ \\ Jeeyun Lee, MD, $P D^{1}$ \\ Joon Oh Park, MD, PhD' \\ Young Suk Park, MD, PhD \\ Ho Yeong Lim, MD, PhD \\ Won Ki Kang, MD, PhD'
}

\section{${ }^{1}$ Division of Hematology-Oncology,}

Department of Medicine, Samsung Medical Center, Sungkyunkwan University School of Medicine, Seoul, ${ }^{2}$ Division of HematologyOncology, Department of Medicine,

Samsung Changwon Hospital,

Sungkyunkwan University School of Medicine Changwon, ${ }^{3}$ Department of Oncology, Asan Medical Center, University of Ulsan College of Medicine, Seoul, ${ }^{4}$ Department of Internal Medicine, Seoul National University Hospital, Cancer Research Institute, Seoul National University College of Medicine, Seoul, ${ }^{5}$ Department of Internal Medicine, Institute for Cancer Research, Yonsei Cancer Center, Yonsei University College of Medicine, Seoul, Korea

\section{Purpose}

Simvastatin has demonstrated anti-tumor activity in preclinical studies via tumor cell senescence, apoptosis, and anti-angiogenesis. This phase II trial evaluated the efficacy and toxicity profile of conventional XELOX and bevacizumab chemotherapy plus simvastatin in metastatic colorectal cancer patients (MCRC).

\section{Materials and Methods}

Patients with MCRC received first-line XELOX in 3-week treatment cycles of intravenous xaliplatin $130 \mathrm{mg} / \mathrm{m}^{2}$ plus bevacizumab $7.5 \mathrm{mg} / \mathrm{kg}$ (day 1), followed by oral capecitabine $1,000 \mathrm{mg} / \mathrm{m}^{2}$ twice daily (day 1-14). Simvastatin $80 \mathrm{mg}$ tablets were taken orally once daily every day during the period of chemotherapy. The primary endpoint was progression-free survival (PFS). Secondary endpoints were response rate, duration of response, overall survival (OS), time to progression, and toxicity.

\section{Results}

From January 2014 to April 2015, 60 patients were enrolled and 55 patients were evaluable for tumor response. The median follow-up duration was 30.1 months (range, 28.5 to 31.7 months). The median PFS was 10.4 months (95\% confidence interval [Cl], 9.6 to 11.1). The median OS of all patients was 19.0 months ( $95 \% \mathrm{Cl}, 11.9$ to 26.0$)$. The disease-control rate and overall response rate were $88.3 \%$ (95\% Cl, 74 to 96$)$ and $58.3 \%(95 \% \mathrm{Cl}, 44$ to 77 ), respectively, by intent-to-treat protocol analysis. There was one complete response and 34 partial responses. One patient experienced grade 3 creatine kinase elevation and liver enzyme elevation.

\section{Conclusion}

Based on the current study, the addition of $80 \mathrm{mg}$ simvastatin to XELOX and bevacizumab showed comparable clinical efficacy in patients with MCRC as first-line chemotherapy and did not increase toxicity.
Correspondence: Won Ki Kang MD, PhD Division of Hematology-Oncology, Department of Medicine, Samsung Medical Center, Sungkyunkwan University School of Medicine, 81 Irwon-ro, Gangnam-gu, Seoul 06351, Korea

Tel: 82-2-3410-1779

Fax: 82-2-3410-1754

E-mail:wkkang@skku.edu
Key words

Colorectal neoplasms, Simvastatin, XELOX, Bevacizumab 


\section{Introduction}

Colorectal cancer is the second most deadly cancer worldwide and is the third and second most commonly diagnosed cancer in males and females, respectively [1]. The treatment of metastatic colorectal cancer (MCRC) has evolved significantly over the decades. The addition of oxaliplatin to oral capecitabine monotherapy led to higher response rates (RRs) and time-to-progression (TTP) in both first- and second-line treatment of advanced colorectal cancer [2,3].

Angiogenesis and maintenance of the tumor vasculature are essential for cancer growth, and vascular endothelial growth factor (VEGF) is essential for both tumor angiogenesis and development [4]. Given the key role of VEGF in angiogenesis, bevacizumab, a humanized monoclonal immunoglobulin G1 antibody targeting circulating VEGF, has been shown to improve RR and survival when combined with any of the first-line and second-line standard cytotoxic chemotherapy regimens in patients with MCRC $[5,6]$. Bevacizumab plus double chemotherapy has become a standard treatment for patients with MCRC. However, limited efficacy and resistance remain outstanding problems $[7,8]$.

Statins are widely prescribed cholesterol-lowering drugs in the primary and secondary prevention of cardiovascular disease with a favorable safety profile [9]. In addition to cholesterol reduction, the anti-proliferative effects of statins on different cancer cell lines have been demonstrated in numerous in vitro studies and in clinical data [10]. Statins act by reducing cholesterol synthesis through the inhibition of 3-hydroxy-3-methylglutayl coenzyme A reductase, the ratelimiting enzyme of the mevalonate pathway [9]. Mevalonate production is reduced, along with its downstream products involved in carcinogenesis, farnesyl pyrophosphate (FPP) and geranylgeranyl pyrophosphate (GGPP) $[9,11]$. FPP and GGPP are essential substrates for posttranslational modifications of the Ras and Rho homolog gene family, member A (RHOA), which plays an important role in the intracellular signal transduction that is responsible for cell growth, proliferation, migration, and survival $[11,12]$. Thus, RHOA gene mutations have been implicated in various cancer types via aberrant cell signaling. Statins have been shown to stabilize the cyclin-dependent kinase inhibitors p21 and p27, thus inhibiting cell signaling pathways involved in metastatic properties of invasive cancers [13]. Based on the effects of statins on posttranscriptional modifications of RAS and RHOA, the anti-tumor effect of statins has been proposed in various cancers, including melanomas, adenocarcinomas, and neuroblastoma [14-16].

In our recent in vitro study, the addition of simvastatin to bevacizumab reduced proliferation, migration, invasion, and tumor formation of endothelial cells. Moreover, we found that colorectal cancer cell media to which simvastatin combined with bevacizumab was added inhibited endothelial cell invasion and was associated with reduced levels of mediators of angiogenesis such as angiopoietin 2, BiP, and heat shock protein $90 \alpha$. Treatment with bevacizumab and simvastatin reduced the growth of xenograft tumors more than bevacizumab alone [17]. We previously reported a phase II trial of simvastatin plus FOLFIRI (irinotecan, 5-fluorouracil, and leucovorin) chemotherapy in MCRC patients and demonstrated the safety of adding simvastatin to chemotherapy [18]. Accordingly, we planned this study to investigate the synergistic effect of simvastatin combined with standard chemotherapy (XELOX [capecitabine plus oxaliplatin] plus bevacizumab) as first-line chemotherapy in patients with MCRC.

\section{Materials and Methods}

\section{Phase II study design}

This was an open-label, single-arm, phase II study conducted at four centers (Samsung Medical Center, Asan Medical Center, Seoul National University Hospital, and Yonsei Cancer Center) in South Korea (ClinicalTrials.gov identifier; NCT02026583). The primary endpoint was progression-free survival (PFS), defined as the time from the start of treatment to progression or death, as assessed by the investigator. Secondary endpoints were independently reviewed $R R$ and overall survival (OS), defined as the time from treatment initiation to death or safety.

\section{Patient eligibility}

Patients aged $\geq 20$ years with histologically confirmed metastatic or recurrent colorectal cancer (CRC), an Eastern Cooperative Oncology Group performance status $\leq 1$, and a life expectancy $>3$ months were enrolled. All patients had to have at least one measurable lesion according to the Response Evaluation Criteria in Solid Tumors (RECIST, ver. 1.1) [19]. Adjuvant chemotherapy, if administered, needed to have been completed at least 12 months before study entry. No previous chemotherapy for metastatic or advanced CRC was permitted, nor was previous exposure to bevacizumab. Patients had to have adequate hematological (absolute neutrophil count $\geq 1.5 \times 10^{9} / \mathrm{L}$; platelet count $\geq 100 \times 10^{9} / \mathrm{L}$; hemoglobin $\geq 10 \mathrm{~g} / \mathrm{dL}$ ), hepatic (total bilirubin $\leq 1.5 \times$ the upper limit of normal [ULN]; alanine aminotransferase and aspartate aminotransferase $\leq 2.5 \times \mathrm{ULN}$, or $\leq 5 \times \mathrm{ULN}$ in the case of hepatic metastases; alkaline phosphatase $\leq 2.5 \times \mathrm{ULN}$, or 
$\leq 5 \times \mathrm{ULN}$ in the case of hepatic or $\leq 10 \times \mathrm{ULN}$ osseous metastases, respectively), and renal function (creatinine clearance by Cockroft formula $\geq 50 \mathrm{~mL} / \mathrm{min}$ or creatinine $\leq 1.5 \mathrm{mg} / \mathrm{dL}$ ).

Key exclusion criteria were prior statin therapy within 1 year from the date of study entry, prior treatment with another anti-VEGF or anti-angiogenic tyrosine kinase inhibitor, prior chemotherapy for MCRC (adjuvant chemotherapy for CRC was permitted), surgery within 28 days of starting treatment, planned radiotherapy for underlying disease (completed radiotherapy in 4 weeks was allowed), and history of malignancy other than MCRC. Patients with uncontrolled hypertension; clinically significant cardiovascular disease, hemorrhagic diathesis, or coagulopathy; use of full-dose anticoagulants or thrombolytics; serious nonhealing wounds; or other medications predisposing to gastrointestinal (GI) ulceration were also excluded.

\section{Treatment}

Patients received intravenous oxaliplatin $130 \mathrm{mg} / \mathrm{m}^{2}$ on day 1 , then oral capecitabine $1,000 \mathrm{mg} / \mathrm{m}^{2}$ twice daily from the evening of day 1 to the morning of day 15 , followed by a 7-day treatment-free interval, repeated in a 3-week cycle. Bevacizumab $7.5 \mathrm{mg} / \mathrm{kg}$ was administered intravenously as a 30- to 90-minute intravenous infusion before oxaliplatin on day 1 of each cycle. Simvastatin was taken orally in an $80 \mathrm{mg}$ tablet once per day every day during the period of chemotherapy. Treatment cycles were repeated every 3 weeks until evidence of disease progression, unacceptable toxicity, or patient refusal. Simvastatin was stopped upon termination of XELOX plus bevacizumab chemotherapy.

Administration of oxaliplatin or capecitabine was delayed as long as there was neutropenia less than $1,500 / \mathrm{mm}^{3}$ or thrombocytopenia less than $75,000 / \mathrm{mm}^{3}$. The dose of oxaliplatin was reduced by $25 \%$ for grade 3 or 4 thrombocytopenia, grade 4 neutropenia, and for paresthesia with pain or functional impairment $>7$ days. In general, for nonhematologic toxicities greater than or equal to Common Terminology Criteria for Adverse Events 4.0 (CTCAE 4.0) grade 3 and considered related to study treatment, treatment was delayed until resolution to grade 1 or to the patient's baseline value before treatment. However, exceptions were possible for grade 2 neurotoxicity. The investigator and patient could decide to continue treatment at a reduced dose with no delay required, as neurotoxicity was not expected to resolve to baseline values in all cases. Dose reductions at the start of the subsequent cycle were based on maximum non-hematologic toxicities from the dose administered in the preceding cycle. In case of grade 3 or 4 neurotoxicity, oxaliplatin was discontinued, and only capecitabine, bevacizumab, and simvastatin were continued. Bevacizumab doses were not reduced or escalated; in cases of serious bevacizumab-related toxicity, bevacizumab was temporarily or permanently suspended. When bevacizumab was discontinued, continuation of XELOX plus simvastatin was considered.

\section{Study assessments}

Pre-study screening assessments included a full medical history, vital signs and physical measurements, and hematologic and blood chemistry tests. Tumor assessments were performed by computed tomography scan, X-ray, and / or magnetic resonance imaging during screening and every two cycles of treatment until disease progression or withdrawal from study medication. In patients whose disease had not progressed when treatment was stopped, tumor assessments were performed every 3 months until progression. In addition to investigator assessment, tumor imagery was evaluated by an independent review committee (IRC), using $\mathrm{X}$-ray, magnetic resonance imaging, or computed tomography scans. Investigators and the IRC assessed the same scans. In addition, survival was monitored at intervals of every 3 months in each patient leaving the study. Tumor response was assessed according to RECIST ver. 1.1 criteria and confirmed at least 6 weeks later by the same evaluation. TTP was defined as the interval between the first dose of study treatment and the first recording of disease progression or death.

Toxicity was graded according to the CTCAE ver. 4.0. In addition, the incidence of the following specific adverse events (irrespective of their possible relationship to bevacizumab treatment) was assessed: GI perforation, woundhealing complications, bleeding, hypertension, proteinuria, and thromboembolism (venous and arterial).

\section{Statistical considerations}

In a phase III trial of bevacizumab combined with oxaliplatin-based chemotherapy as first-line therapy, median PFS was 9.4 months in the bevacizumab group and 8.0 months in the placebo group [8]. Based on those data, the median PFS of $\mathrm{H} 0$ was assumed as 9 months, and the median PFS of $\mathrm{H} 1$ as 13 months. Using a one-sided alpha at 0.05 and a power of $80 \%, 54$ patients were needed for the trial. With consideration for dropouts and ineligibility of $10 \%$, a total of 60 patients were needed. The efficacy analyses were based on the intent-to-treat population. The primary endpoint was PFS, as assessed by the investigators. Secondary endpoints were disease control rate, overall response rate, OS, and toxicity. Kaplan-Meier estimates were used in the analysis of timeto-event variables, and the 95\% confidence interval (CI) for the median time to event was computed. Safety was analyzed in all patients who received at least one dose of study medication. Descriptive statistics are reported as proportions and medians. Clinical cutoff for the study analysis was Feb- 
Table 1. Baseline patient characteristics

\begin{tabular}{|c|c|}
\hline Characteristic & No. $(\%)(\mathrm{n}=60)$ \\
\hline Age, median (range, yr) & $57(27-74)$ \\
\hline \multicolumn{2}{|l|}{ Sex } \\
\hline Male & $35(58.3)$ \\
\hline Female & $25(41.7)$ \\
\hline \multicolumn{2}{|l|}{ ECOG PS } \\
\hline 0 & $15(25.0)$ \\
\hline 1 & $45(75.0)$ \\
\hline \multicolumn{2}{|l|}{ Histology of adenocarcinoma } \\
\hline Well differentiated & $16(26.7)$ \\
\hline Moderately differentiated & $37(61.7)$ \\
\hline Mucinous adenocarcinoma & $1(1.7)$ \\
\hline Poorly differentiated & $6(10.0)$ \\
\hline \multicolumn{2}{|l|}{ B-Raf status } \\
\hline Wild type & $45(75.0)$ \\
\hline Mutated & $8(13.3)$ \\
\hline Unknown & $7(11.7)$ \\
\hline \multicolumn{2}{|l|}{ K-Ras status } \\
\hline Wild type & $39(65.0)$ \\
\hline Mutated & $16(26.7)$ \\
\hline Unknown & $5(8.3)$ \\
\hline \multicolumn{2}{|l|}{ Localization of the primary tumor } \\
\hline Right side colon & $15(25.0)$ \\
\hline Left side colon & $45(75.0)$ \\
\hline \multicolumn{2}{|l|}{ State at study enrollment } \\
\hline Recurrence & $20(33.3)$ \\
\hline Locally advanced and distant metastasis & $40(66.7)$ \\
\hline \multicolumn{2}{|l|}{ Site of distant metastasis } \\
\hline Liver & $49(81.7)$ \\
\hline Lung & $22(36.7)$ \\
\hline Lymph nodes & $16(26.7)$ \\
\hline Peritoneal seeding & $8(13.3)$ \\
\hline Bone & $3(5.0)$ \\
\hline
\end{tabular}

ECOG PS, Eastern Cooperative Oncology Group performance status.

ruary $28,2017$.

\section{Ethical statement}

The study was conducted in accordance with the World Medical Association Declaration of Helsinki and the Ethical Guidelines for Clinical Studies. All patients gave their written informed consent, conforming to institutional guidelines, indicating that they were aware of the investigational nature of the study. This study was reviewed and approved by the institutional review board (IRB) of the participating institutions (IRB No. 2013-07-088).

\section{Results}

\section{Patient characteristics and treatment}

From January 2014 to April 2015, 60 patients were enrolled. Baseline characteristics are summarized in Table 1. The median age was 57 years (range, 24 to 74 years). Approximately one-third of the patients had recurrent disease after curative resection. Tumors were located in the cecum in three, ascending colon in 11, transverse colon in one, descending colon in four, sigmoid colon in 18 , rectosigmoid colon in nine, and rectum in 14 patients. Collectively, 15 patients $(25 \%)$ had a tumor in a proximal (from the cecum to transverse colon) location, and 45 patients $(75 \%)$ had a tumor in a distal location.

Patients ( $n=60)$ had adenocarcinoma that was well, moderate, or poorly differentiated in 16 (26.7\%), 37 (61.7\%), and six $(10.0 \%)$ of cases, respectively. Metastases were found in the liver in 49 cases $(81.7 \%)$, the lung in 22 (36.7\%), lymph nodes in $16(26.7 \%)$, peritoneal seeding in eight $(13.3 \%)$, bone in three $(5.0 \%)$, and at two or more sites in $32(53.3 \%)$.

\section{Efficacy}

In total, 689 cycles were administered with a median of nine cycles per patient (range, 1 to 45 cycles). The median follow-up duration was 30.1 months (range, 29.2 to 33.4 months). The median PFS was 10.4 months (95\% CI, 9.6 to 11.1) (Fig. 1A), and median OS was 19.0 months (95\% CI, 13.1 to 24.7 ) (Fig. 1B). Fifty-five of 60 patients (91.6\%) were assessable for response. The disease-control rate and overall response rate were $88.3 \%$ (95\% CI, 74 to 96$)$ and $58.3 \%$ (95\% CI, 44 to 77 ), respectively, by intent-to-treat protocol analysis. There was one complete response (CR) and 34 partial responses (PRs). All patients were included in the survival analysis. Two patients are currently still in the trial, and 58 patients discontinued study medication. The main reasons for discontinuation were disease progression $(n=43,74.1 \%)$ and patient refusal $(n=7,12.0 \%)$ (Table 2). Two patients wanted to stop after confirming CR or PR, and one patient underwent surgery after PR.

\section{Toxicity}

Table 3 details adverse events leading to treatment discontinuation and predefined adverse events of special interest in response to the statin. Most adverse events were mild to moderate, the most common being sensory neuropathy $(48 \%)$, nausea $(37 \%)$, fatigue or weakness $(33 \%)$, and hand foot syndrome (32\%). Significant toxicity (grade 3 or 4 ) presented as nausea (5\%), neutropenia (5\%), fatigue $(2 \%)$, rash 
A

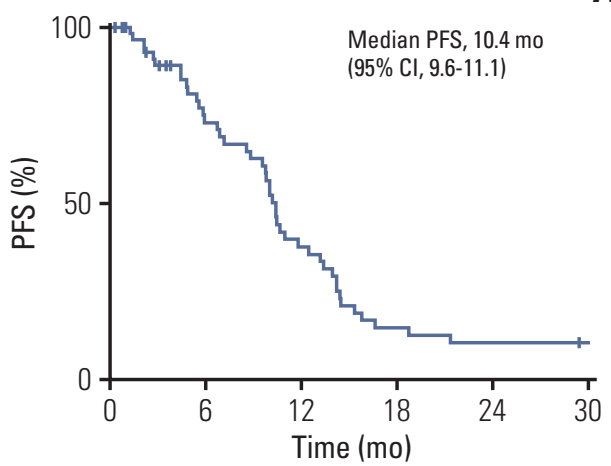

Fig. 1. Progression-free survival (PFS) (A) and overall survival (OS) (B) with simvastatin plus XELOX and bevacizumab in metastatic colorectal cancer patients.

Table 2. Best response and reasons for discontinuation

\begin{tabular}{|lc|}
\hline Best response $(\mathbf{n}=60)$ & No. $(\%)$ \\
\hline Complete response & $1(1.7)$ \\
\hline Partial response & $34(56.7)$ \\
\hline Stable disease & $18(30.0)$ \\
\hline Progression & $2(3.3)$ \\
\hline Not evaluable & $5(8.3)$ \\
\hline Reasons for discontinuation $(\mathbf{n}=58)$ & \\
\hline Progression & $43(74.1)$ \\
\hline Consent withdrawal & $7(12.0)$ \\
\hline Follow up loss & $3(5.2)$ \\
\hline Severe adverse event ${ }^{\text {a) }}$ & $2(3.5)$ \\
\hline Wait and see after CR or PR & $2(3.5)$ \\
\hline Operation after PR & $1(1.7)$ \\
\hline
\end{tabular}

$\mathrm{CR}$, complete response; PR, partial response. ${ }^{a)}$ Bowel perforation occurred after one cycle in two patients.

$(2 \%)$, stomatitis $(2 \%)$, hand foot syndrome $(2 \%)$, vomiting $(2 \%)$, and diarrhea $(2 \%)$. Two patients experienced grade 3 creatine kinase $(\mathrm{CK})$ elevation. One patient experienced grade $3 \mathrm{CK}$ elevation and liver enzyme elevation, suggesting statin-induced myopathy; treatment was stopped. The other one seemed to experience grade $3 \mathrm{CK}$ elevation without liver enzyme elevation as also statin related toxicity. Bowel perforation occurred in two patients after the first cycle of treatment.
Table 3. Toxicity profile

\begin{tabular}{lcl} 
Toxicity & Grade 1-2 & Grade 3-4 \\
Non-hematologic toxicities & & \\
$\quad$ Anorexia & $11(18.3)$ & 0 \\
Fatigue & $20(33.3)$ & $1(1.7)$ \\
Rash & $9(15.0)$ & $1(1.7)$ \\
Hyperpigmentation & $17(28.3)$ & 0 \\
Stomatitis & $7(11.7)$ & $1(1.7)$ \\
Hand foot syndrome & $19(31.7)$ & $1(1.7)$ \\
Dry skin & $15(25.0)$ & 0 \\
Nausea & $22(36.7)$ & $3(5.0)$ \\
Vomiting & $2(3.3)$ & $1(1.7)$ \\
Diarrhea & $1(1.7)$ & $1(1.7)$ \\
Constipation & $10(16.7)$ & 0 \\
\hline Bowel perforation & 0 & $2(3.3)$ \\
Peripheral neuropathy & $29(48.3)$ & 0 \\
\hline Increased CK & $2(3.3)$ & $2(3.3)$ \\
Increased AST / ALT & $1(1.7)$ & $1(1.7)$ \\
Hematologic toxicities & & \\
Neutropenia & $10(16.7)$ & $3(5.0)$ \\
\hline Thrombocytopenia & $3(5.0)$ & 0 \\
\hline
\end{tabular}

Values are presented as number (\%). CK, cytokeratin; AST, aspartate aminotransferase; ALT, alanine transaminase.

\section{Discussion}

This was a single-arm, phase II study to evaluate the efficacy and safety of the addition of simvastatin, a synthetic 3-hydroxy-3-methyglutaryl coenzyme A reductase inhibitor, to XELOX and bevacizumab as first-line chemotherapy in MCRC. The median PFS was 10.1 months, which was not a 
significantly superior outcome compared to previous studies. Our observed median OS (19.0 months; 95\% CI, 13.1 to 24.7) was similar to that observed in a prior trial in MCRC (XELOX plus bevacizumab) [8].

Preclinical studies continue to report the tremendous potential of simvastatin in cancer cell lines through inhibition of cell proliferation, promotion of apoptosis, promotion of tumor cell differentiation, modulation of the tumor microenvironment, and immunomodulation. Statins have been investigated for a variety of cancers, and there are also several ongoing trials of statin treatment in various cancers, including in our group. Combination therapy clinical trials with statins and other chemotherapeutic agents have shown encouraging results. Previously, our group conducted an open-label, phase II trial evaluating the efficacy and toxicity profile of conventional FOLFIRI chemotherapy in combination with simvastatin in patients with MCRC and found that this combination exhibited promising anti-tumor activity [18]. In that study, the median survival of all patients was 21.8 months (95\% CI, 14.4 to 29.2), and the median TTP was 9.9 months (95\% CI, 6.4 to 13.3). However, in this study, the PFS and OS of statin plus bevacizumab did not reach statistical significance. Given the promising results reported with statin use in various cancers, novel combination treatments with statins warrant further studies to explore and validate potential synergies in combinations. Further research is also needed to determine which subtypes of patients benefit from statin therapy, how statins may potentiate other anti-cancer approaches, and the appropriate dosing schedule to use.

The anti-cancer effects of statins result from inhibition of endothelial cell proliferation and migration, as well as induction of apoptosis. Our previous experimental data suggested simvastatin had anti-angiogenic effect and potentiated the effect of bevacizumab in vitro and in vivo [17]. In one study, statin is associated with induced apoptosis of microvascular endothelial cells and lowered VEGF serum levels implicating a possible anti-angiogenic role in cancer treatment [20-23]. Statin can induce apoptosis (high dose), senescence (low dose), and antiangiogenic effect in various cancer cells, though the toxicity of statin is not significant. However, a limitation in using statins for cancer therapy is that the optimal dose and schedule of statin treatment have not been validated. Therefore it is worthy of further clinical study with addition of statin to conventional chemotherapeutic agents in cancer patients.

In terms of toxicity, two patients experienced GI perforation after 1 cycle. In previous randomized controlled trials, GI perforation occurred in $1 \%-2 \%$ of patients treated with bevacizumab for MCRC. The majority of GI perforation events $(26 / 37)$ occurred $\leq 6$ months after starting bevacizumab (median, 3.35 months) [24]. Compared to the incidence, treatment with the statin plus bevacizumab did not increase GI perforation risk. The results showed comparable clinical efficacy in MCRC patients as first-line chemotherapy without increased toxicity.

In conclusion, the addition of $80 \mathrm{mg}$ simvastatin to XELOX and bevacizumab showed comparable clinical efficacy in patients with MCRC as first-line chemotherapy and did not increase toxicity. In the future, we hope further studies will be performed to investigate efficient combination strategies involving statins and cytotoxic chemotherapy regimens.

\section{Conflicts of Interest}

Simvastatin was provided by CJ and bevacizumab by Roche. Neither company was involved in the collection or analysis of the data or in the preparation of the manuscript.

\section{Acknowledgments}

We thank all participating patients and their families, as well as the research nurses and study coordinators.

\section{References}

1. Siegel RL, Miller KD, Fedewa SA, Ahnen DJ, Meester RG, Barzi A, et al. Colorectal cancer statistics, 2017. CA Cancer J Clin. 2017;67:177-93.

2. Matsui T, Nagata N, Hirata K, Okazaki S, Sato S, Nakamura $\mathrm{M}$, et al. Bi-weekly capecitabine-oxaliplatin (XELOX) plus bevacizumab as first-line treatment of metastatic colorectal cancer: the PHOENiX Trial. Anticancer Res. 2016;36:3437-43.

3. Lee JJ, Chu E. An update on treatment advances for the firstline therapy of metastatic colorectal cancer. Cancer J. 2007;13: 276-81.
4. Carmeliet P. VEGF as a key mediator of angiogenesis in cancer. Oncology. 2005;69 Suppl 3:4-10.

5. Giantonio BJ, Catalano PJ, Meropol NJ, O'Dwyer PJ, Mitchell $\mathrm{EP}$, Alberts SR, et al. Bevacizumab in combination with oxaliplatin, fluorouracil, and leucovorin (FOLFOX4) for previously treated metastatic colorectal cancer: results from the Eastern Cooperative Oncology Group Study E3200. J Clin Oncol. 2007; 25:1539-44.

6. Grothey A, Sugrue MM, Purdie DM, Dong W, Sargent D, Hedrick E, et al. Bevacizumab beyond first progression is 
associated with prolonged overall survival in metastatic colorectal cancer: results from a large observational cohort study (BRiTE). J Clin Oncol. 2008;26:5326-34.

7. Hurwitz H, Fehrenbacher L, Novotny W, Cartwright T, Hainsworth J, Heim W, et al. Bevacizumab plus irinotecan, fluorouracil, and leucovorin for metastatic colorectal cancer. N Engl J Med. 2004;350:2335-42.

8. Saltz LB, Clarke S, Diaz-Rubio E, Scheithauer W, Figer A, Wong R, et al. Bevacizumab in combination with oxaliplatinbased chemotherapy as first-line therapy in metastatic colorectal cancer: a randomized phase III study. J Clin Oncol. 2008;26:2013-9.

9. Goldstein JL, Brown MS. Regulation of the mevalonate pathway. Nature. 1990;343:425-30.

10. Osmak M. Statins and cancer: current and future prospects. Cancer Lett. 2012;324:1-12.

11. Kubatka P, Kruzliak P, Rotrekl V, Jelinkova S, Mladosievicova B. Statins in oncological research: from experimental studies to clinical practice. Crit Rev Oncol Hematol. 2014;92:296-311.

12. Chae YK, Yousaf M, Malecek MK, Carneiro B, Chandra S, Kaplan J, et al. Statins as anti-cancer therapy; Can we translate preclinical and epidemiologic data into clinical benefit? Discov Med. 2015;20:413-27.

13. Denoyelle C, Vasse M, Korner M, Mishal Z, Ganne F, Vannier $\mathrm{JP}$, et al. Cerivastatin, an inhibitor of HMG-CoA reductase, inhibits the signaling pathways involved in the invasiveness and metastatic properties of highly invasive breast cancer cell lines: an in vitro study. Carcinogenesis. 2001;22:1139-48.

14. Keyomarsi K, Sandoval L, Band V, Pardee AB. Synchronization of tumor and normal cells from G1 to multiple cell cycles by lovastatin. Cancer Res. 1991;51:3602-9.

15. Prasanna P, Thibault A, Liu L, Samid D. Lipid metabolism as a target for brain cancer therapy: synergistic activity of lovastatin and sodium phenylacetate against human glioma cells. J Neurochem. 1996;66:710-6.

16. Jakobisiak M, Bruno S, Skierski JS, Darzynkiewicz Z. Cell cycle-specific effects of lovastatin. Proc Natl Acad Sci U S A. 1991;88:3628-32.

17. Lee SJ, Lee I, Lee J, Park C, Kang WK. Statins, 3-hydroxy- 3-methylglutaryl coenzyme A reductase inhibitors, potentiate the anti-angiogenic effects of bevacizumab by suppressing angiopoietin2, BiP, and Hsp90alpha in human colorectal cancer. Br J Cancer. 2014;111:497-505.

18. Lee J, Jung KH, Park YS, Ahn JB, Shin SJ, Im SA, et al. Simvastatin plus irinotecan, 5-fluorouracil, and leucovorin (FOLFIRI) as first-line chemotherapy in metastatic colorectal patients: a multicenter phase II study. Cancer Chemother Pharmacol. 2009;64:657-63.

19. Therasse P, Arbuck SG, Eisenhauer EA, Wanders J, Kaplan RS, Rubinstein L, et al. New guidelines to evaluate the response to treatment in solid tumors. European Organization for Research and Treatment of Cancer, National Cancer Institute of the United States, National Cancer Institute of Canada. J Natl Cancer Inst. 2000;92:205-16.

20. Negre-Aminou P, van Vliet AK, van Erck M, van Thiel GC, van Leeuwen RE, Cohen LH. Inhibition of proliferation of human smooth muscle cells by various HMG-CoA reductase inhibitors; comparison with other human cell types. Biochim Biophys Acta. 1997;1345:259-68.

21. Pirillo A, Jacoviello C, Longoni C, Radaelli A, Catapano AL. Simvastatin modulates the heat shock response and cytotoxicity mediated by oxidized LDL in cultured human endothelial smooth muscle cells. Biochem Biophys Res Commun. 1997; 231:437-41.

22. Schaefer CA, Kuhlmann CR, Gast C, Weiterer S, Li F, Most $\mathrm{AK}$, et al. Statins prevent oxidized low-density lipoproteinand lysophosphatidylcholine-induced proliferation of human endothelial cells. Vascul Pharmacol. 2004;41:67-73.

23. Alber HF, Dulak J, Frick M, Dichtl W, Schwarzacher SP, Pachinger $\mathrm{O}$, et al. Atorvastatin decreases vascular endothelial growth factor in patients with coronary artery disease. J Am Coll Cardiol. 2002;39:1951-5.

24. Kabbinavar FF, Flynn PJ, Kozloff M, Ashby MA, Sing A, Barr $\mathrm{CE}$, et al. Gastrointestinal perforation associated with bevacizumab use in metastatic colorectal cancer: results from a large treatment observational cohort study. Eur J Cancer. 2012;48:1126-32. 